\title{
Smad Ubiquitination Regulatory Factor 1 (Smurf1) Promotes Thyroid Cancer Cell Proliferation and Migration via Ubiquitin- Dependent Degradation of Kisspeptin-1
}

\author{
Chunyan Yan ${ }^{a}$ Haiying Su ${ }^{b}$ Xiyuan Song ${ }^{a} \quad$ Huiling Cao ${ }^{a}$ Lingling Kong ${ }^{a}$ \\ Wen Cui ${ }^{\mathrm{a}}$
}

aDepartment of Pathology, College of Basic Medicine, Jining Medical University, Jining, bepartment of Gynecology, Jining No. 1 People's Hospital, Jining, China

\section{Key Words}

Thyroid cancer $\cdot$ Smad ubiquitination regulatory factor $1 \cdot$ Kisspeptin-1 $\bullet$ Ubiquitin-dependent degradation $• \mathrm{NF}-\mathrm{KB}$ signaling pathway

\begin{abstract}
Background/Aims: Thyroid cancer is the most common malignancy in human endocrine system. Smad ubiquitination regulatory factor 1 (Smurf1) is an E3 ubiquitin-protein ligase in ubiquitin-proteasome pathway (UPP) system. This study aimed to investigate the effects of Smurf1 on thyroid cancer proliferation and metastasis, as well as underlying potential mechanism. Methods: The expression levels of Smurf1 in thyroid tumor tissues and thyroid cancer cells were detected by western blotting and qRT-PCR. Then, the effects of upregulation or down-regulation of Smurf1 on thyroid cancer cell viability, migration, invasion, proliferation and apoptosis were measured using trypan blue exclusion assay, two-chamber migration (invasion) assay, cell colony formation assay and Guava Nexin assay, respectively. The ubiquitination of kisspeptin-1 (KISS-1) was assessed by protein ubiquitination assay. Finally, the effects of KISS-1 overexpression on activity of nuclear factor-kappa B (NF-KB) signaling pathway, as well as thyroid cancer cell viability, migration, invasion, proliferation and apoptosis were also detected, respectively. Results: Smurf1 was highly expressed in thyroid tumor tissues and thyroid cancer cells. Up-regulation of Smurf1 promoted the viability, migration, invasion and proliferation of thyroid cancer cells. Knockdown of Smurf1 had opposite effects. Moreover, smurf1 promoted the ubiquitination of KISS-1. Overexpression of KISS-1 inactivated NF-kB pathway, suppressed thyroid cancer cell viability, migration, invasion and proliferation, and induced cell apoptosis. Conclusion: Up-regulation of Smurf1 exerted important roles in thyroid cancer formation and development by promoting thyroid cancer proliferation and metastasis. The ubiquitin-dependent degradation of KISS-1 induced by Smurf1 and the
\end{abstract}


activation of NF-KB signaling pathway might be involved in this process. Smurf1 could be an effective therapy target and biomarker for thyroid cancer treatment.

(C) 2018 The Author(s)

Published by S. Karger AG, Basel

\section{Introduction}

Thyroid cancer is the most common malignancy in human endocrine system [1]. According to different clinical features, thyroid cancer can be classified as papillary thyroid cancer (PTC, 80\%), follicular thyroid cancer (FTC, 10-15\%), poorly differentiated thyroid cancer (PDTC, 2-3\%), anaplastic thyroid cancer (ATC, 1-2\%) and thyroid squamous cell carcinoma (SCC, 1-2\%) [2]. The incidence of thyroid cancer has remarkably increased in recent decades [3]. Numerous factors contribute to the canceration of normal thyroid cells, including DNA mutation and methylation, change of gene expression and activation of intracellular signaling pathways $[2,4]$. It is urgently needed to identify effective therapy targets and biomarkers for thyroid cancer treatment.

Ubiquitin-proteasome pathway (UPP) consists of ubiquitin (Ub), ubiquitin-activating enzyme (E1), ubiquitin-binding enzymes (E2s), ubiquitin-protein ligases (E3s), 26S proteasome, ubiquitin dissociation enzymes (DUBs) and plays critical regulatory roles in cell proliferation and apoptosis by degradation of proteins [5]. A number of studies have demonstrated that the abnormal activation of UPP lead to the canceration of normal cells through aberrant degradation of tumor suppressor proteins [6, 7]. Smad ubiquitination regulatory factor 1 (Smurf1) is an E3 ubiquitin-protein ligase, which participants in recruiting ubiquitin-loaded E2s and recognizing specific substrates [8]. Kwon et al. proved that Smurf1 played an important role in epidermal growth factor (EGF)-induced breast cancer cell migration and invasion [9]. Ke et al. pointed out that Smurf1 promoted growth and metastasis of clear cell renal cell carcinoma by activation of extracellular signal-regulated kinase/ribosomal S6 kinase 1 (ERK/RSK1) and phosphatidylinositol 3 kinase/protein kinase $\mathrm{B} /$ mammalian target of rapamycin (PI3K/AKT/mTOR) pathways [10]. However, the effects of Smurf1 on thyroid cancer cell proliferation and metastasis remain unclear.

Kisspeptin-1 (KISS-1), initially described as a tumor metastasis suppressor gene in melanoma, encodes KISS-1 protein and further processes to a several of peptides, including kisspeptin-10, kisspeptin-13, kisspeptin-14, and kisspeptin-54 in cells [11, 12]. These peptides are endogenous ligands of G protein-coupled receptor, which play central roles in the regulation of cell growth and death $[13,14]$. Down-regulation of KISS-1 has been proved in a variety of cancer cells, including thyroid cancer $[14,15]$. Gao et al. demonstrated that the absence or down-regulation of KISS-1 was involved in the thyroid tumorigenesis, invasion and metastasis [16].

In this study, we focused our investigation on the regulatory roles of Smurf1 in thyroid cancer cell viability, migration, colony formation and apoptosis. The ubiquitin-dependent degradation of KISS-1 induced by Smurf1 was also investigated. These findings will be helpful for identifying the roles of Smurf1 in thyroid cancer development and providing effective therapy targets and biomarkers for thyroid cancer treatment.

\section{Materials and Methods}

\section{Patients and tissue specimens}

Clinical tissue specimens were obtained from 3 patients diagnosed with PTC ( 2 women and 1 man, $52.26 \pm 14.83$ years old), 1 patient diagnosed with SCC (man, 55 years old) and 1 patient diagnosed with FTC (women, 61 year old). None of the patients received any therapeutic before surgery. Informed consents were obtained from each patient before the experiments performed. The thyroid tumor tissues and the corresponding normal adjacent thyroid tissues were surgically resected and collected in the separate tubes. This study was approved by the Institutional Ethics Committee of the Jining No. 1 People's Hospital. 


\section{Cellular Physiology Cell Physiol Biochem 2018;49:2047-2059 \begin{tabular}{ll|l} 
and BiOChemistry Published online: 22 September, 2018 & $\begin{array}{l}\text { (c) } 2018 \text { The Author(s). Published by S. Karger AG, Basel } \\
\text { www.karger.com/cpb }\end{array}$ \\
\hline
\end{tabular}}

Yan et al.: Roles of Smurf1 in Thyroid Cancer Cells

Cell culture

Human SCC cell line SW579, Human PTC cell lines TPC-1, K1, BCPAP and human thyroid cell lines HTori3, Nthy-ori3 were all purchased from American Type Culture Collection (Manassas, VA, USA). SW579, TPC-1, K1 and BCPAP cells were cultured in L-15 medium (Sigma-Aldrich, MO, USA). HT-ori3 and Nthy-ori3 cells were cultured in RPMI-1640 medium (Sigma-Aldrich). Mediums were supplemented with $10 \%$ fetal calf serum (FCS, Life Science, UT, USA), $100 \mathrm{U} / \mathrm{ml}$ penicillin (Sigma-Aldrich), $100 \mu \mathrm{g} / \mathrm{ml}$ streptomycin (Life Science) and $2 \mathrm{mM}$ glutamine (Sigma-Aldrich). Cultures were maintained at $37^{\circ} \mathrm{C}$ with $5 \% \mathrm{CO}_{2}$ in a humidity atmosphere.

\section{Quantitative Reverse Transcription PCR ( $q R T-P C R$ )}

Total RNAs in cells was extracted using TRIzol reagent (Life Technologies Corporation, CA, USA) supplemented with DNaseI (Sigma-Aldrich) according to the manufacturer's instructions. Reverse transcription was performed using Multiscribe RT kit (Qiagen, Hilden, Germany). The reverse transcription condition was set at $10 \mathrm{~min}$ at $25^{\circ} \mathrm{C}, 30 \mathrm{~min}$ at $48^{\circ} \mathrm{C}$, and a final step of $5 \mathrm{~min}$ at $95^{\circ} \mathrm{C}$. Primes were synthesized as follows: Smurf1 forward primer: 5'-AGTTCGTGGCCAAA TAGTGG-'3, reverse primer: 5'-GTTCCTTCGTTCTCCAGCAG-'3; KISS-1 forward primer: 5'-AGCTGCTGCTTCTCCTCTGT-'3, reverse primer: 5'-AGGCTTGCT CTCTGCATACC-'3; GAPDH forward primer: 5'-CTCGCTTCGGCAGCACA-'3, reverse primer: 5'-AACGCTTCACGAATTTGCGT-'3.

\section{Transfection and generation of stably transfected cell lines}

Short-hairpin RNA directed against Smurf1 was ligated into the U6/GFP/Neo plasmid (GenePharma, Shanghai, China) and referred as sh-Smurf1. The full-length Smurf1 or KISS-1 sequences were constructed in pcDNA3.1 plasmids and referred as pc-Smurf1 or pc-KISS-1, respectively. The empty pcDNA3.1 plasmid and U6/GFP/Neo plasmid carrying non-targeting Smurf1 sequence were acted as negative controls and referred as pcDNA3.1 and shNC, respectively. Smurf1-FLAG and KISS-1-FLAG expression plasmids were constructed as previously described [17]. SW579 or TPC-1 cells $\left(1 \times 10^{5}\right)$ were seeded into 96-well plates (Coring Inc., NY, USA) for overnight and then transfected in line with the manufacturer's protocol by using lipofectamine 3000 reagent (Invitrogen, CA, USA). The stably transfected cells were selected using the culture medium

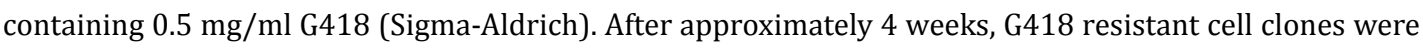
established. qRT-PCR was performed to verify transfection efficiency.

\section{Cell viability assay}

Cell viability was detected using trypan blue exclusion assay. Briefly, after relevant transfection according to the experiments design, cells $\left(1 \times 10^{5}\right)$ were seeded into 24 -well plates (Coring Inc.) and incubated for $24 \mathrm{~h}$. Then, cells were harvested and stained with $0.4 \%$ trypan blue solution. The viable cells were counted using cell counting chamber. Cell viability (\%) was calculated by numbers of non-stained cells/numbers of total cells $\times 100 \%$.

\section{Cell migration assay}

Cell migration was measured using a two-chamber migration assay with a pore size of $8 \mathrm{~mm}$ (Millipore, Bedford, MA, USA) as previously described [18]. The number of migrated cells were observed using microscope (Nikon, Japan). Cell migration (\%) was calculated by numbers of migrated cells in transfection group/numbers of migrated cells in control group $\times 100 \%$.

\section{Cell invasion assay}

Cell invasion assay was detected similarly with the cell migration assay, except that the transwell membrane was pre-treated with Matrigel (BD Bioscience, Franklin Lakes, NJ, USA). Briefly, after relevant transfection, $2 \times 10^{3}$ cells were suspended into $200 \mu \mathrm{l}$ serum-free culture medium and added into the upper chamber. Complete culture medium $(600 \mu \mathrm{l})$ was added into the lower chamber. After incubation at $37^{\circ} \mathrm{C}$ for $48 \mathrm{~h}$, cells were fixed with methanol immediately. Non-invaded cells in upper chamber were removed using cotton swab carefully. Invaded cells in lower chamber were stained using crystal violet solution (Beyotime Biotechnology, Shanghai, China) and counted under Microscope (Nikon, Japan). Cell invasion (\%) was calculated by numbers of invaded cells in transfection group/numbers of invaded cells in control group $\times$ $100 \%$. 


\section{Cellular Physiology Cell Physiol Biochem 2018;49:2047-2059 \begin{tabular}{l|l|l} 
and Biochemistry 10.1159/000493715 & $\begin{array}{l}\text { C } 2018 \text { The Author(s). Published by S. Karger AG, Basel } \\
\text { www.karger.com/cpb }\end{array}$ \\
\hline
\end{tabular}}

\section{Cell colony formation assay}

After relevant transfection, cells were harvested and diluted to a density of $1 \times 10^{4}$ cells $/ \mathrm{ml}$. Then, 1 $\times 10^{3}$ cells were plated into 6 - $\mathrm{cm}$ cell culture dishes and incubated in L-15 medium containing $10 \%$ serum at $37^{\circ} \mathrm{C}$. Two to three weeks later, cell clones were fixed with $4 \%$ paraformaldehyde and stained with $0.1 \%$ crystal violet solution. The relative colony formation number (\%) was calculated by numbers of colony in transfection group/numbers of colony in control group $\times 100 \%$.

\section{Cell apoptosis assay}

Cell apoptosis was identified by Guava Nexin Assay kit (Guava Technologies, CA, USA) according to the manufacturer's instruction, which utilizes Annexin V-PE to detect the phosphatidylserine on the membrane of apoptotic cells. After relevant transfection, a total of $1 \times 10^{5}$ cells were seeded into 24-cell plates (Coring Inc.) and incubated for $24 \mathrm{~h}$. Then, all cells were harvested, washed with PBS for three times. After that, 100 $\mu \mathrm{l}$ cells of each group were mixed with $100 \mu \mathrm{l}$ nexin assay kit solution for $20 \mathrm{~min}$ at $37^{\circ} \mathrm{C}$ in the dark. Cell apoptosis was detected using flow cytometer analysis (Millipore). Data was analyzed using FCS Express software (De Novo Soft, CA, USA).

\section{Immunoprecipitation assay}

After relevant treatment, SW579 and TPC-1 cells were washed with PBS for three times, and then lysed in Western/Immunoprecipitation lysis buffer (Beyotime Biotechnology, Jiangsu, China). Cell lysates containing total proteins were centrifuged at $14000 \mathrm{~g}$ for $15 \mathrm{~min}$. After pre-clearing with $50 \mu \mathrm{L}$ protein A/G-Sepharose (Invitrogen, CA, USA) for $1 \mathrm{~h}$, the supernatants were mixed with $5 \mu \mathrm{g}$ anti-FLAG or anti-Myc (Cell Signaling Technology, MA, USA, cat number: 8146 or 2276) cross-linked to protein A/G-Sepharose bead and incubated at $4^{\circ} \mathrm{C}$ overnight. After that, beads were washed with lysis buffer for three times and re-suspended into sodium dodecyl sulfate (SDS) loading buffer. Western blotting was done using relevant antibodies

\section{Protein ubiquitination assay}

The protein ubiquitination assay was performed as reported previously [19]. SW579 or TPC-1 cells were treated with MG132 (25 $\mu$ M, Sigma-Aldrich) for $5 \mathrm{~h}$ before harvest to avoid the proteasome-mediated degradation. Then cell lysate was prepared in HEPES lysis buffer containing with protease inhibitors, and proteins were immune-precipitated with indicated antibody and measured by western blotting. E1, E2, HAUb, Smurf1-Glutathione-S-Transferase (GST) and KISS-FLAG were incubated at $30^{\circ} \mathrm{C}$ for $2 \mathrm{~h}$, and the assay was terminated with protein loading buffer.

\section{$N F-\kappa B$ activity assay}

SW579 and TPC-1 cells were co-transfected with pc-KISS-1 and NF- $\kappa B$ signal reporter vector (Qiagen), which contains a mix of a vector with the firefly luciferase gene controlled by an NF- $\kappa$ B responsive promoter. After incubation, luciferase activity was detected using Dual-Glo Luciferase Reporter kit (Promega, Madison, WI, USA) following the manufacturer's instruction.

\section{Western blotting assay}

The total proteins in tissues and cells were extracted using RIPA lysis buffer (Beyotime Biotechnology) supplemented with protease inhibitor (Roche, Basel, Switzerland) and quantified using BCA ${ }^{\mathrm{TM}}$ Protein Assay Kit (Pierce, WI, USA). Western blotting was established using Bio-Rad Bis-Tris Gel system following the manufacturer's protocol and performed as previously described [20]. Anti-Bcl2-associated X (Bax, 2772), anti-Cleaved-Caspase 3 (9661), anti-Cleaved-Caspase 9 (9501), anti-GAPDH (2118) FLAG (8146), Myc (2278), Ub (3933) and HA (3724) were obtained from Cell Signaling Technology (MA, USA). Anti-KISS-1 (sc101246), anti-p-p65 (sc-52401), anti-p65 (sc-56735), anti-p-Inhibitor of nuclear factor-kappa B (NF-кB)

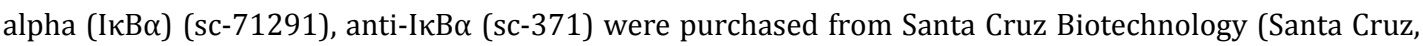
CA, USA). Protein bands were captured using ECL Plus western blotting detection kit (Amersham Pharmacia Biotech, Germany). 


\section{Cellular Physiology Cell Physiol Biochem 2018;49:2047-2059 \begin{tabular}{ll|l} 
and Biochemistry Published online: 22 September, 2018 & $\begin{array}{l}\text { (c) } 2018 \text { The Author(s). Published by S. Karger AG, Basel } \\
\text { www.karger.com/cpb }\end{array}$ \\
\hline
\end{tabular}

\section{Statistical analysis}

All above experiments were performed in triplicate and the values were reported as mean \pm standard deviation (SD). SPSS 19.0 statistical software (SPSS Inc., IL, USA) was used for statistical analysis. P-values were calculated using a One-Way analysis of Variance (ANOVA). A P-value of $<0.05$ was considered to be statistically significant.

\section{Results}

Smurf1 was highly expressed in thyroid tumor tissues and thyroid cancer cells

The expression levels of Smurf1 in thyroid tumor tissues and thyroid cancer cells were measured using western blotting and qRT-PCR, respectively. As shown in Fig. 1A, Smurf1 was highly expressed in thyroid tumor tissues compared to the corresponding normal adjacent thyroid tissues. Moreover, the protein and mRNA levels of Smurf1 in human thyroid cancer SW579, TPC-1, K1 and BCPAP cells were higher than in human normal thyroid HT-ori3 and Nthy-ori3 cells (Fig. $1 \mathrm{~B}, \mathrm{P}<0.05, \mathrm{P}<0.01$ or $\mathrm{P}<0.001$ ). These results implied that the upregulation of Smurf1 might be involved in the tumorigenesis of thyroid normal cells.

Smurf1 participated in the regulation of thyroid cancer SW579 and TPC-1 cell viability, migration, proliferation and apoptosis

To detect the effects of Smurf on thyroid cancer cell viability, migration, invasion, proliferation and apoptosis, pc-Smurf1 and sh-Smurf1 were transfected into SW579 and TPC-1 cells, respectively. Results showed that transfection with pc-Smurf1 increased the mRNA and protein levels of Smurf1 and transfection with sh-Smurf1 decreased the mRNA and protein levels of Smurf1 in SW579 and TPC- 1 cells (Fig. 2A, $\mathrm{P}<0.01$ or $\mathrm{P}<0.001$ in mRNA level). Viability of SW579 and TPC-1 cells was both noticeably enhanced after transfection

Fig. 1. Smurf1 was highly expressed in thyroid tumor tissues and thyroid cancer cells. (A) The protein expression levels of Smurf1 in five cases of thyroid tumor tissues and their corresponding normal adjacent tissues were detected using western blotting. (B) The protein and mRNA expression levels of Smurf1 in human thyroid cancer cell lines SW579, TPC-1, K1, BCPAP and human thyroid cell lines HT-ori3, Nthy-ori3 were measured using western blotting and qRT-PCR, respectively. Data was expressed as means \pm SD of three independent experiments. Smurf1: Smad Ubiquitination Regulatory Factor 1; PTC: Papillary thyroid cancer; SCC: Thyroid squamous cell carcinoma; FTC: follicular thyroid cancer; $\mathrm{N}$ : normal tissue; T: tumor tissue. ${ }^{* *} \mathrm{P}<0.01,{ }^{* * *} \mathrm{P}<0.001$ versus HT-ori3 cells; ${ }^{\#} \mathrm{P}<0.05,{ }^{\#} \mathrm{P}<0.01$, ${ }^{\# \# \# P<0.001}$ versus Nthy-ori3-1 cells.

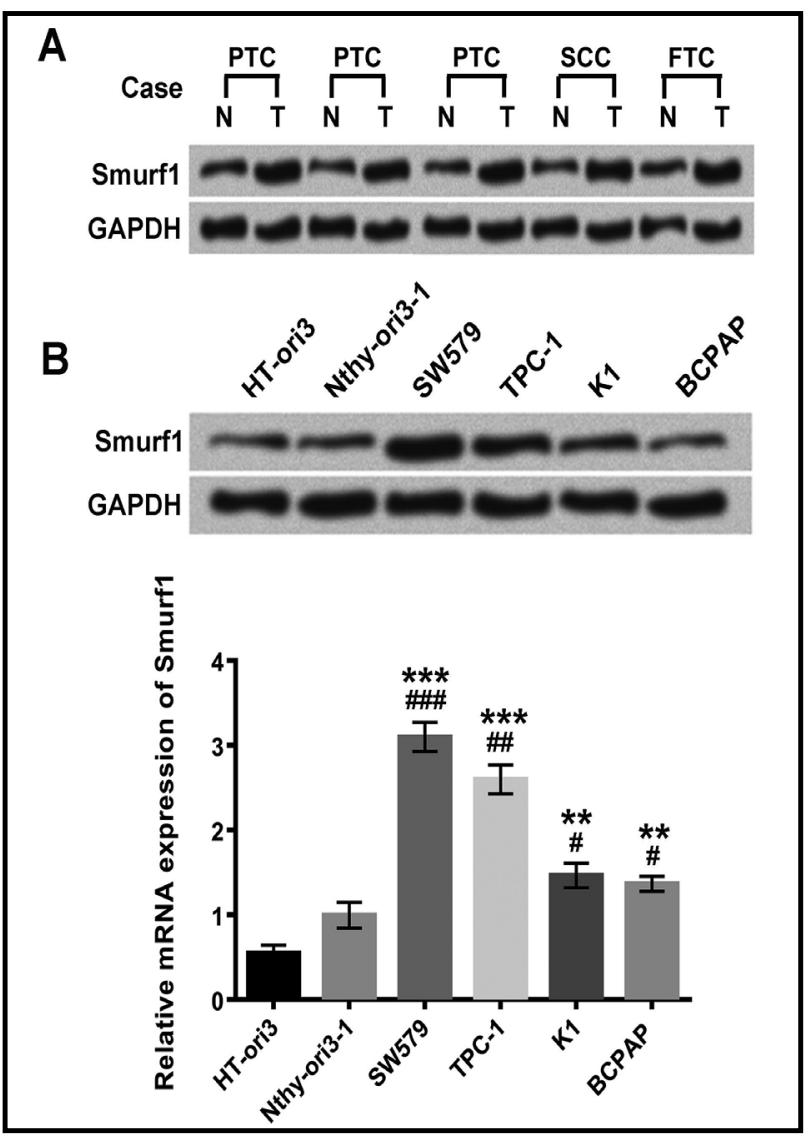




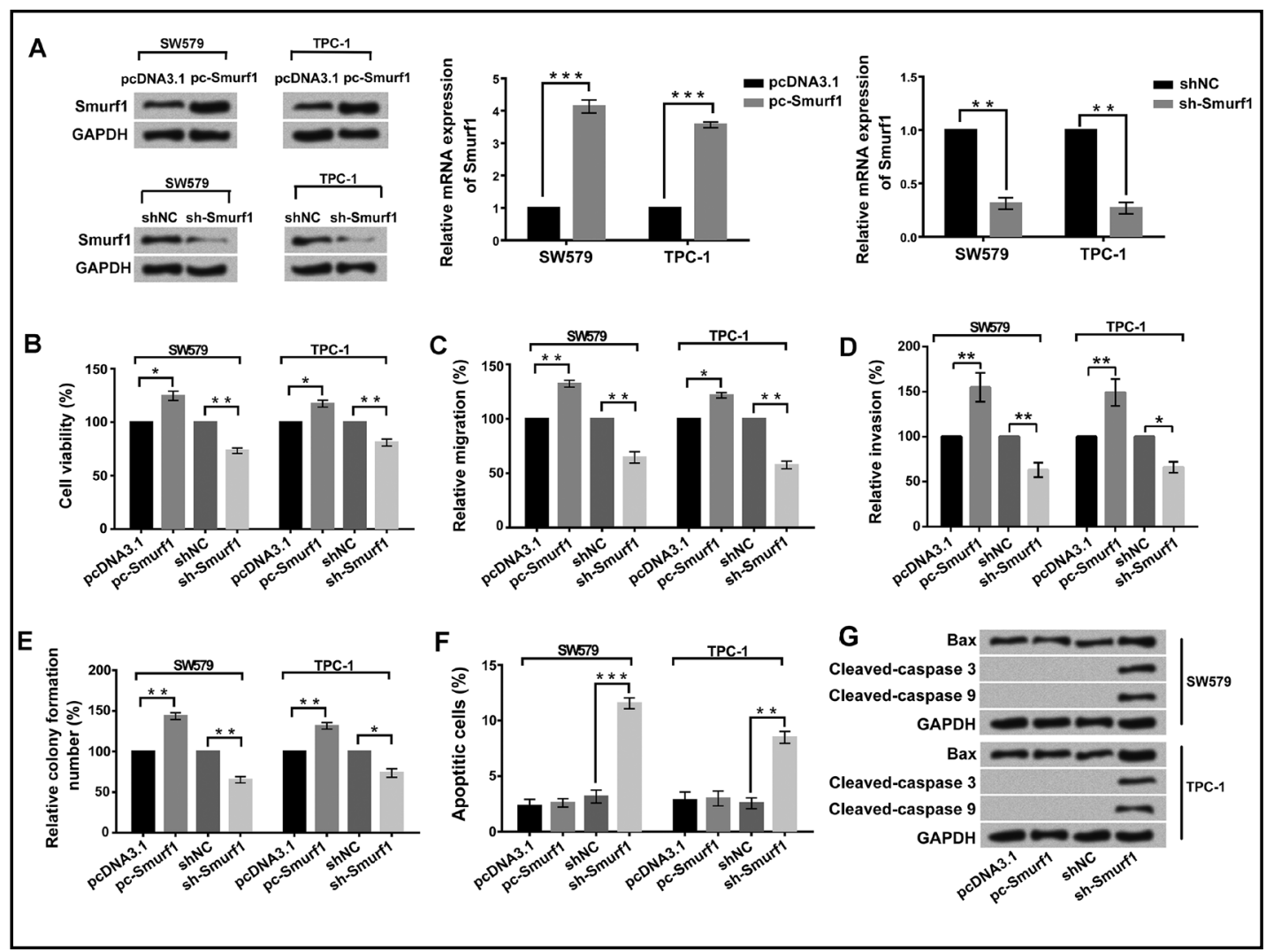

Fig. 2. Smurf1 participated in the regulation of thyroid cancer SW579 and TPC-1 cell viability, migration, colony formation and apoptosis. (A) The protein and mRNA expression levels of Smurf1 in SW79 and TPC-1 cells after transfection with pc-Smurf1 or sh-Smurf1 were detected by western blotting and qRTPCR, respectively. (B) Viability, (C) Migration, (D) Invasion and (E) Relative colony formation number of SW579 and TPC-1 cells after transfection with pc-Smurf1 or sh-Smurf1 were measured by trypan blue exclusion assay, two-chamber migration assay, two-chamber invasion assay and cell colony formation assay, respectively. ( $F$ and G) Flow cytometer analysis and western blotting were performed to evaluate the cell apoptosis and protein expression levels of Bax, Cleaved-Caspase 3, Cleaved-Caspase 9 after transfection with pc-Smurf1 or sh-Smurf1. Data was expressed as means \pm SD of three independent experiments. Smurf1: Smad Ubiquitination Regulatory Factor 1; Bax: Bcl2-associated $\mathrm{X} .{ }^{*} \mathrm{P}<0.05,{ }^{* *} \mathrm{P}<0.01,{ }^{* * *} \mathrm{P}<0.001$.

with pc-Smurf1, but obviously reduced after transfection with sh-Smurf1 (Fig. 2B, P $<0.05$ or $\mathrm{P}<0.01$ ). After transfection with pc-Smurf1, the relative migration and invasion of SW579 and TPC-1 cells were significantly increased (Fig. $2 \mathrm{C}$ and $2 \mathrm{D}, \mathrm{P}<0.05$ or $\mathrm{P}<0.01$ ). Transfection with sh-Smurf1 had opposite effects. In addition, the relative colony formation numbers of SW579 and TPC-1 cells were markedly increased by transfection with pc-Smurf1 and decreased by transfection with sh-Smurf1 (Fig. $2 \mathrm{E}, \mathrm{P}<0.05$ or $\mathrm{P}<0.01$ ). For cell apoptosis, as shown in Fig. 2F, transfection with sh-Smurf1 remarkably increased the rates of apoptosis of SW579 and TPC- 1 cells $(\mathrm{P}<0.01$ or $\mathrm{P}<0.001)$. However, transfection with pc-Smurf1 had no effects on SW579 and TPC-1 cell apoptosis. These results were further verified by western blotting, which pointed out that the protein expression levels of Bax, Cleaved-Caspase 3, Cleaved-Caspase 9 in SW579 and TPC-1 cells were enhanced by sh-Smurf1 transfection (Fig. 2G). These results suggested that overexpression of Smurf1 promoted SW579 and TPC-1 cell viability, migration, invasion and proliferation, while suppression of Smurf1 had opposite effects and induced cell apoptosis. 


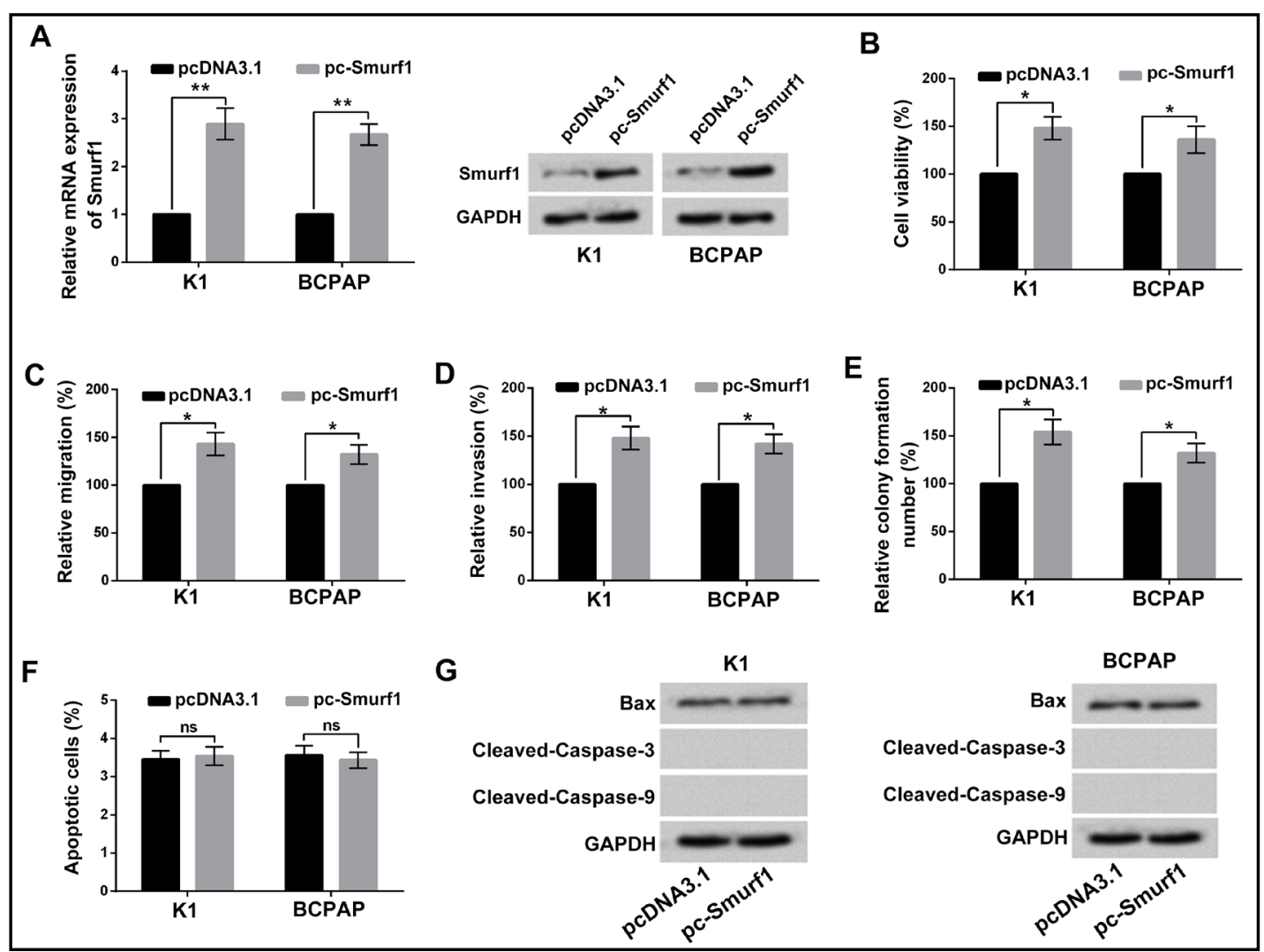

Fig. 3. Overexpression of Smurf1 promoted K1 and BCPAP cell viability, migration, invasion and proliferation. After pc-Smurf1 transfection, (A) The mRNA and protein levels of Smurf1 in K1 and BCPAP cells, (B-F) The viability, migration, invasion, colony formation and apoptosis of K1 and BCPAP cells, and (G) The expression levels of Bax, Cleaved-Caspase- 3 and Cleaved-Caspase- 9 in K1 and BCPAP cells were detected using qRTPCR, western blotting, trypan blue exclusion assay, two-chamber migration assay, two-chamber invasion assay and cell colony formation assay, respectively after transfection with pc-Smurf1. Data was expressed as means \pm SD of three independent experiments. Smurf1: Smad Ubiquitination Regulatory Factor 1; Bax: Bcl2-associated $\mathrm{X} .{ }^{*} \mathrm{P}<0.05,{ }^{* *} \mathrm{P}<0.01$.

Overexpression of Smurf1 promoted K1 and BCPAP cell viability, migration, invasion and proliferation

To further explore the effects of Smurf1 on thyroid cancer K1 and BCPAP cell viability, migration, invasion, proliferation and apoptosis, pc-Smurf1 was transfected into K1 and BCPAP cells, respectively. Fig. $3 \mathrm{~A}$ displayed that pc-Smurf1 transfection remarkably upregulated the mRNA and protein expression levels of Smurf1 in K1 and BCPAP cells $(\mathrm{P}<0.01$ in mRNA level). The results of Fig. 3B-3D presented that pc-Smurf1 transfection obviously enhanced viability, migration and invasion of K1 and BCPAP cells $(\mathrm{P}<0.05)$. Compared to pcDNA3.1 group, the relative colony formation numbers of K1 and BCPAP cells were both increased in pc-Smurf1 transfection group (Fig. 3E, P < 0.05). Fig. 3F showed that pc-Smurf1 transfection had no significant effects on $\mathrm{K} 1$ and BCPAP cell apoptosis. The expression levels of Bax, Cleaved-Caspase- 3 and Cleaved-Caspase- 9 in K1 and BCPAP cells were also not changed after pc-Smurf1 transfection (Fig. 3G). These findings indicated that overexpression of Smurf1 promoted thyroid cancer K1 and BCPAP cell viability, migration, invasion and proliferation, but had no effects on cell apoptosis. 
Smurf1 negatively regulated the protein level of KISS-1 in thyroid cancer cells

The mRNA and protein levels of KISS-1 in SW579 and TPC-1 cells after transfection with pc-Smurf1 or sh-Smurf1 were respectively detected using qRT-PCR and western blotting. Fig. 4A showed that the relative mRNA expression level of KISS-1 in SW579 cells was not change after transfection with pc-Smurf1 or sh-Smurf1. However, the protein level of KISS-1 in SW579 cells was reduced by pc-Smurf1 transfection but enhanced by sh-Smurf1 transfection. Similar results were found in TPC-1 cells (Fig. 4B). These results suggested that Smurf1 negatively regulated the protein level of KISS-1 in thyroid cancer SW579 and TPC-1 cells.

Smurf1 promoted the ubiquitination of KISS-1 in thyroid cancer SW579 and TPC-1 cells

The effect of Smurf1 on KISS-1 ubiquitination was detected by ubiquitination assay. As shown in Fig. 5A, the ubiquitination level of KISS-1-FLAG was increased by treatment with MG132, a proteasome inhibitor, which suggested that the proteasome regulated KISS-1 ubiquitination in thyroid cancer SW579 and TPC-1 cells. Overexpression of Smurf1-FLAG decreased the KISS-1 protein level in a dose-dependent manner in the present of MG132 (Fig. 5B). Moreover, overexpression of Smurf1 enhanced the ubiquitination of KISS-1 (Fig. 5C). In addition, purified Smurf1 protein promoted the ubiquitination of KISS-1 directly in vitro (Fig. 5D). Taken together, these results indicated that Smurf1 promoted the ubiquitination of KISS-1 in thyroid cancer SW579 and TPC-1 cells.

Overexpression of KISS-1 inhibited the NF- $\kappa B$ signaling pathway in thyroid cancer SW579 and TPC-1 cells

pc-KISS-1 was used to increase the relative expression of KISS-1 in SW579 and TPC-1 cells. The results showed that the mRNA levels of KISS-1 in SW579 and TPC-1 cells were significantly increased after pc-KISS- 1 transfection (Fig. $6 \mathrm{~A}, \mathrm{P}<0.01$ or $\mathrm{P}<0.001$ ). The

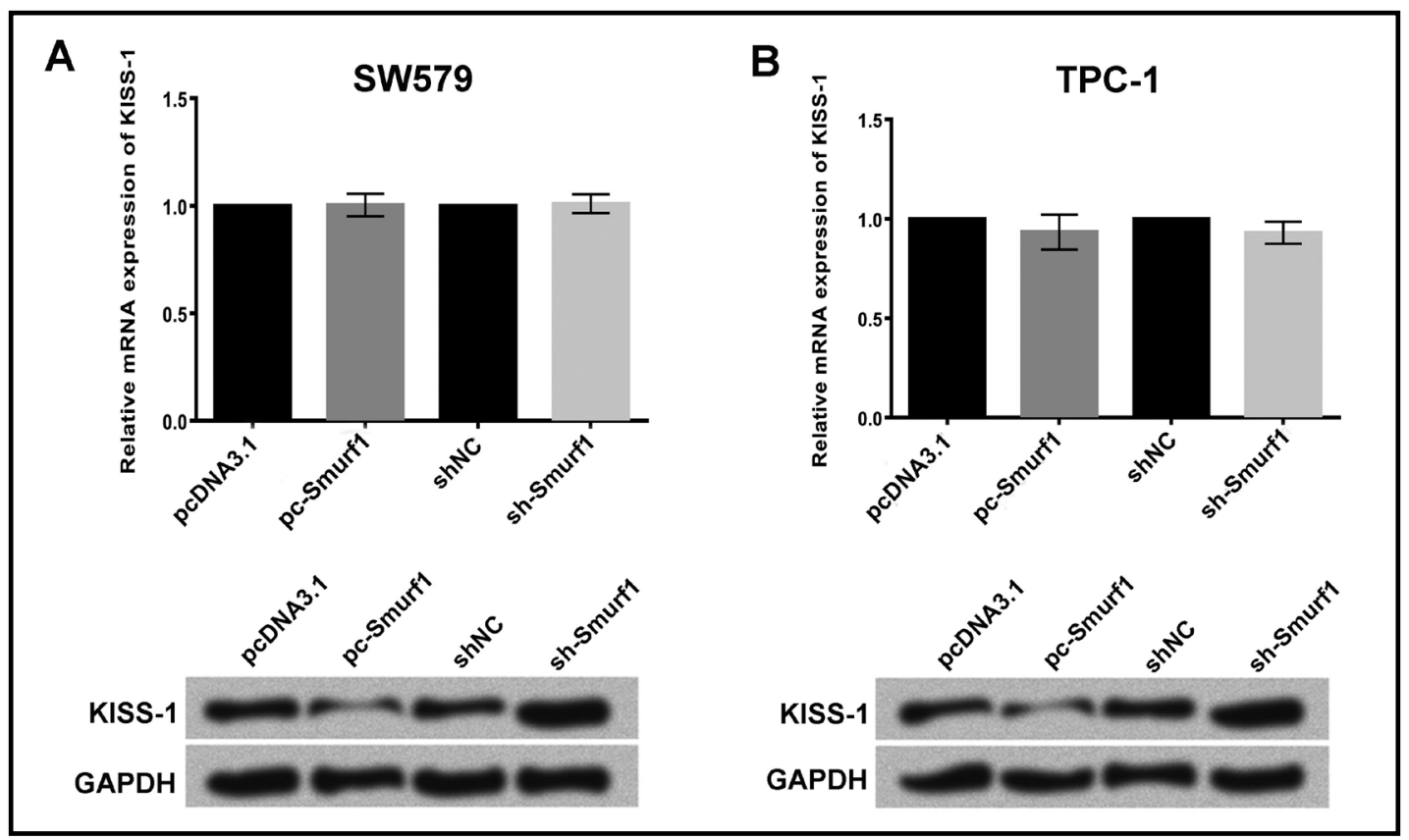

Fig. 4. Smurf1 negatively regulated the protein levels of KISS-1 in thyroid cancer cells. (A) The mRNA and protein levels of KISS-1 after transfection with pc-Smurf1 or sh-Smurf1 in SW579 cells were detected by qRTPCR and western blotting, respectively. (B) The mRNA and protein levels of KISS-1 after transfection with pc-Smurf1 or sh-Smurf1 in TPC-1 cells were detected by qRT-PCR and western blotting, respectively. Data was expressed as means \pm SD of three independent experiments. Smurf1: Smad Ubiquitination Regulatory Factor 1; KISS-1: Kisspeptin-1. 


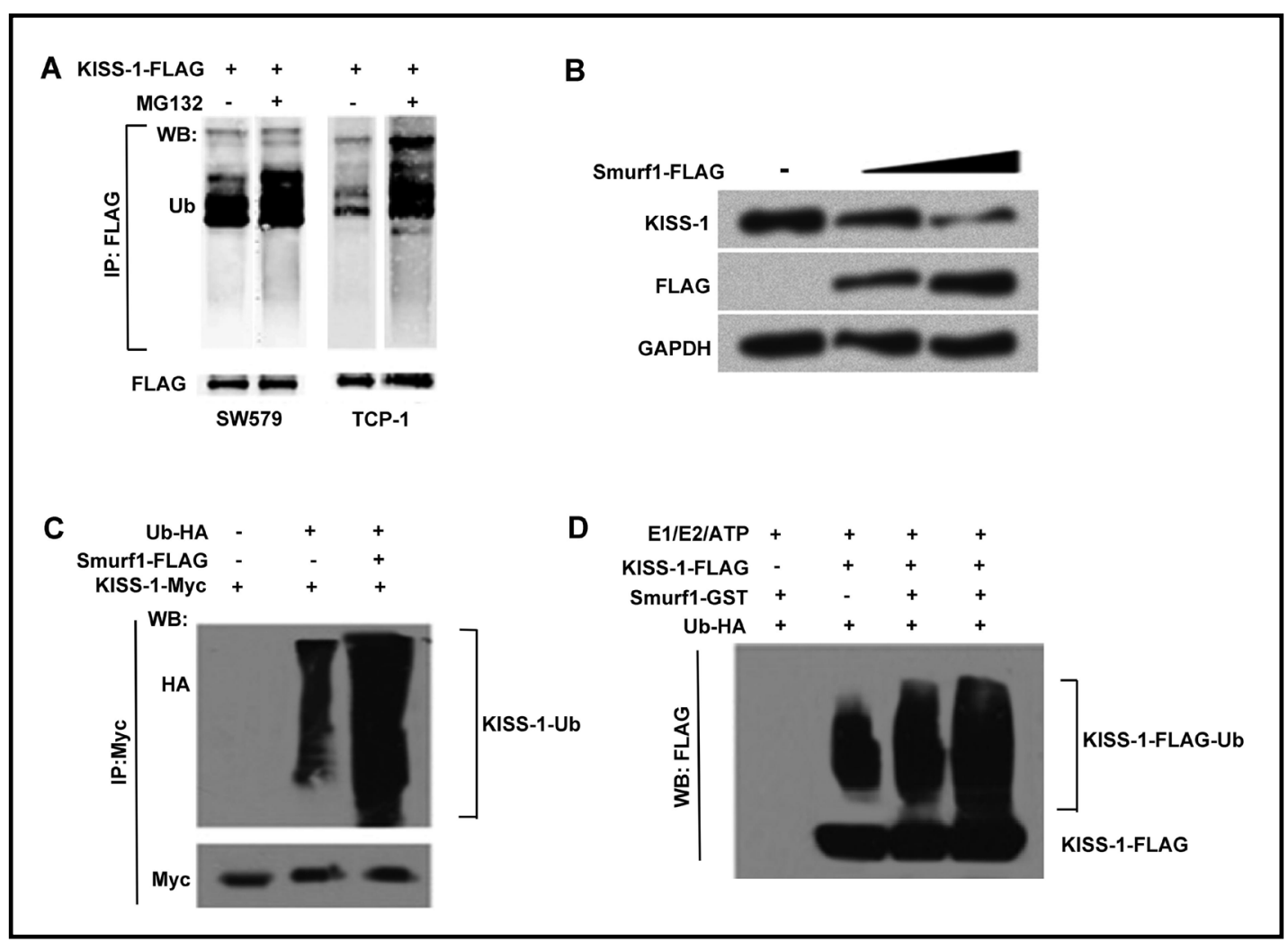

Fig. 5. Smurf1 promoted the ubiquitination of KISS-1 in thyroid cancer SW579 and TPC-1 cells. (A) SW579 and TPC- 1 cells were transfected with KISS-1-FLAG and were treated with or without MG132 $(25 \mu \mathrm{M})$ for $5 \mathrm{~h}$ before harvest. Then KISS-1-FLAG was immunoprecipitated. Western blotting was done using anti-FLAG or anti-Ub antibody. (B) The protein levels of KISS-1 after overexpression of Smurf1-FLAG were evaluated using western blotting. (C) SW579 cells were transfection with Smurf1-FLAG expression plasmids. Then cells were treated with MG132 $(25 \mu \mathrm{M})$ for $5 \mathrm{~h}$ to avoid the proteasome-mediated degradation. After that, cells lysates were harvested and immunoprecipitated with anti-Myc antibody. Western blotting was used to analysis the immunoprecipitates with anti-HA antibody, which indicated the ubiquitination of KISS-1. (D) E1, E2, $\mathrm{HA}-\mathrm{Ub}$, Smurf1-GST and KISS-FLAG were incubated at $30^{\circ} \mathrm{C}$ for $2 \mathrm{~h}$ in ubiquitination buffer. Ubiquitination of KISS-1 was evaluated by western blotting with anti-FLAG antibody. Data was expressed as means \pm SD of three independent experiments. Smurf1: Smad Ubiquitination Regulatory Factor 1; E1: Ubiquitin activating enzyme; E2: Ubiquitin conjugating enzyme; KISS-1: Kisspeptin1; IP: immunoprecipitation; GST: Glutathione-S-Transferase.

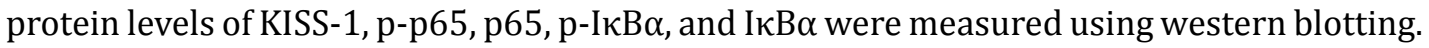
As shown in Fig. 6B, after pc-KISS-1 transfection, the protein levels of KISS-1 in SW579 and TPC-1 cells were both enhanced. Moreover, transfection with pc-KISS-1 down-regulated the expression levels of p-p65 and p-IкB $\alpha$ in both SW579 and TPC- 1 cells. The results of reporter assay also presented that pc-KISS-1 transfection resulted in down-regulation of NF$\kappa \mathrm{B}$ activity in both SW579 and TPC-1 cells (Fig. 6C, P $<0.01$ ). These findings implied that KISS-1 was involved in the regulation of NF- $\mathrm{KB}$ signaling pathway in thyroid cancer SW579 and TPC- 1 cells, and overexpression of KISS- 1 inhibited the activation of NF- $\kappa B$ signaling pathway in thyroid cancer cells.

Overexpression of KISS-1 inhibited SW579 and TPC-1 cell viability, migration, invasion and proliferation, and induced cell apoptosis

Finally, the effects of KISS-1 overexpression on SW579 and TPC-1 cell viability, migration, invasion, proliferation and apoptosis were also analyzed. As presented in Fig. 7A-7D, pc-KISS-1 transfection remarkably suppressed the viability, migration, invasion and colony formation 
of SW579 and TPC-1 cells $(\mathrm{P}<0.05$ or $\mathrm{P}<0.01)$. Fig. $7 \mathrm{E}$ showed that pc-KISS-1 transfection dramatically induced SW579 and TPC-1 cell apoptosis $(\mathrm{P}<0.001)$. Compared to pcDNA3.1 group, the expression levels of Bax, Cleaved-Caspase 3 and Cleaved-Caspase- 9 in SW579 and TPC-1 cells were all increased in pc-KISS-1 transfection group (Fig. 7F). These findings indicated that overexpression of KISS-1 inhibited thyroid cancer SW579 and TPC1 cell viability, migration, invasion and proliferation, and induced cell apoptosis.
Fig. 6. Overexpression of KISS1 inhibited NF- $\mathrm{BB}$ signaling pathway in thyroid cancer SW579 and TPC-1 cells. (A) The relative mRNA expression levels of KISS-1 in SW579 and TPC-1 cells after transfection with pcKISS- 1 were detected using qRTPCR. (B) The protein levels of KISS-1, p-p65, p65, p-ІкB $\alpha$, ІкB $\alpha$ in SW579 and TPC-1 cells after transfection with pc-KISS-1 were evaluated using western blotting. (C) Reporter assay was conducted to measure the activity of NF- $\mathrm{BB}$ in SW579 and TPC- 1 cells after co-transfection with pc-KISS-1 and NF- $\kappa B$ signal reporter vector. Data was expressed as means \pm SD of three independent experiments. KISS-1: Kisspeptin-1; NF-кB: nuclear factor-kappa $B$; І $\kappa \mathrm{B} \alpha$ : Inhibitor of NF- $\mathrm{KB}$ alpha. ${ }^{* *} \mathrm{P}<0.01,{ }^{* * *} \mathrm{P}<0.001$.

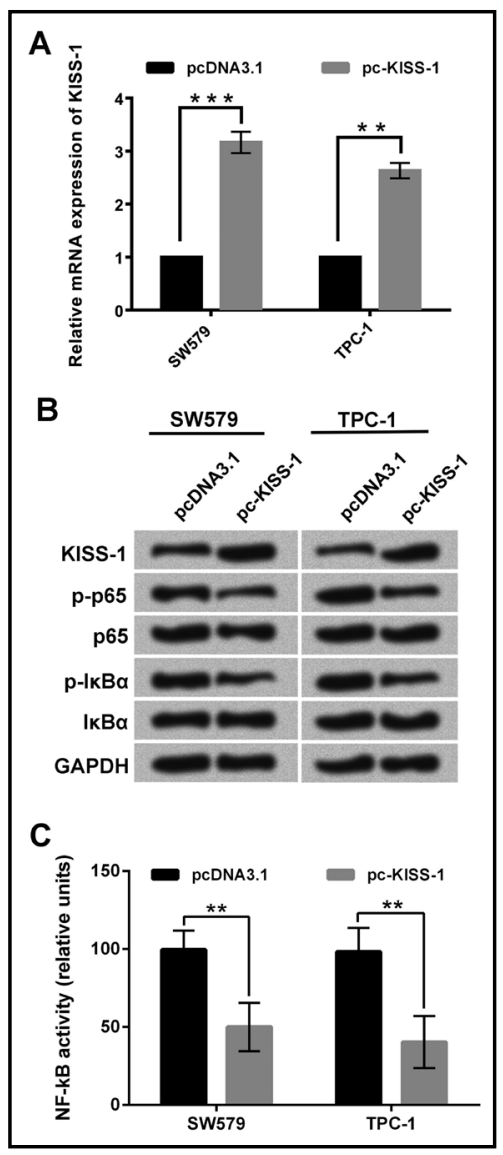

Fig. 7. Overexpression of KISS-1 inhibited SW579 and TPC-1 cell viability, migration, invasion and proliferation, and induced cell apoptosis. After pc-KISS-1 transfection, (A-E) the viability, migration, invasion, colony formation and apoptosis of SW579 and TPC-1 cells, and (F) the expression levels of Bax, Cleaved-Caspase-3 and Cleaved-Caspase- 9 in SW579 and TPC- 1 cells were assessed using trypan blue exclusion assay, two-chamber migration assay, twochamber invasion assay, cell colony formation assay and western blotting, respectively. Data was expressed as means \pm SD of three independent experiments. KISS-1: Kisspeptin-1; Bax: Bcl2-associated $\mathrm{X} . \quad{ }^{*} \mathrm{P}<0.05$,
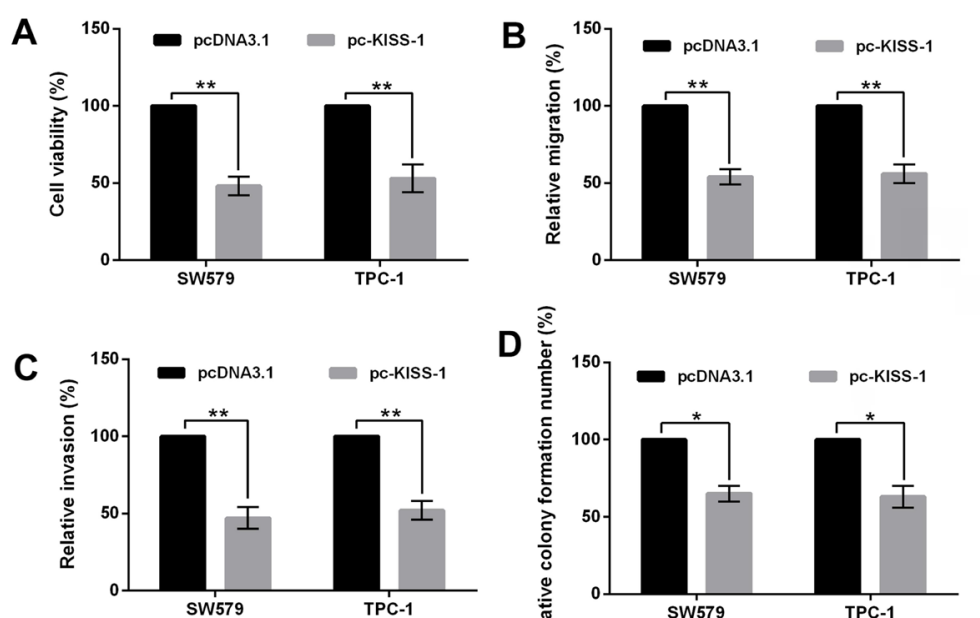

D
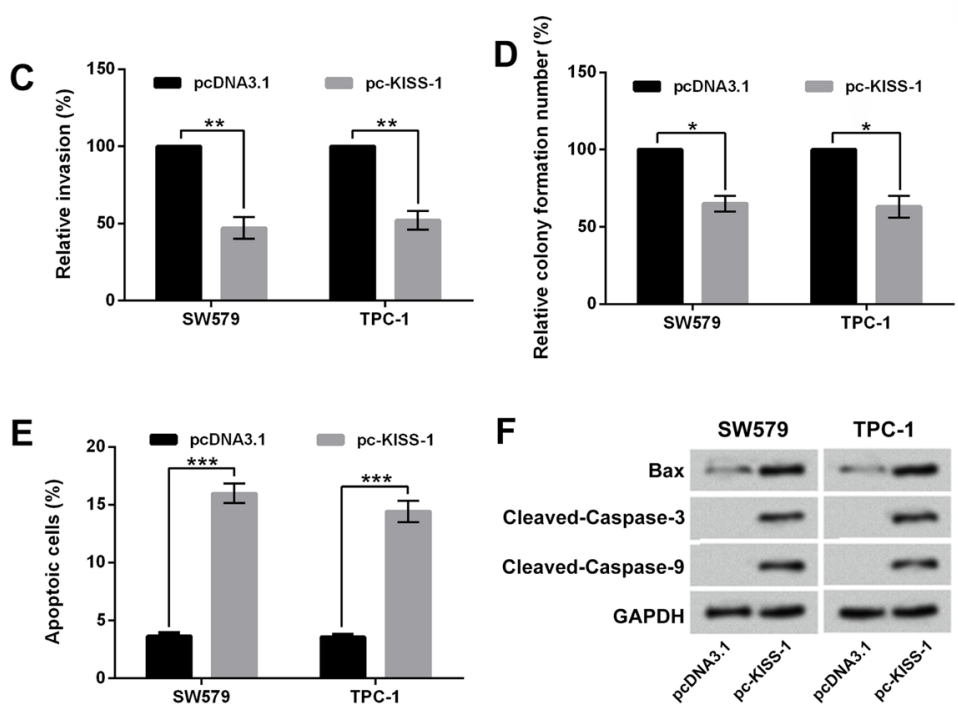

F

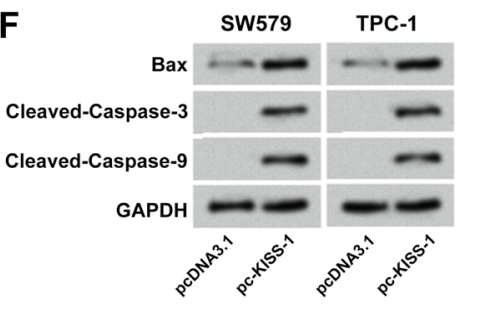




\section{Cellular Physiology Cell Physiol Biochem 2018;49:2047-2059

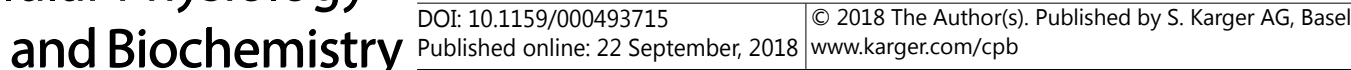 Yan et al.: Roles of Smurf1 in Thyroid Cancer Cells}

\section{Discussion}

UPP mediated protein ubiquitin-dependent degradation plays essential roles in normal cell growth and proliferation [21]. Whereas the function change of UPP contributes to development of various cancers [7]. Researchers have made great efforts to definite the specific effects of UPP on cancer cell proliferation and metastasis. In this research, we investigated the functional roles of Smurf1, an E3 ubiquitin-protein ligase in UPP system, in thyroid cancer cell viability, migration, proliferation and apoptosis. The effects of Smurf1 on ubiquitin-dependent degradation of KISS-1 were also detected. Our results found that Smurf1 was highly expressed in thyroid tumor tissues and thyroid cancer cells. Overexpression of Smurf1 significantly enhanced the viability, migration, invasion and proliferation of thyroid cancer cells. Knockdown of Smurf1 had opposite effects. Moreover, Smurf1 promoted the ubiquitin-dependent degradation of KISS-1 in thyroid cancer cells. Overexpression of KISS1 inactivated NF-кB pathway, suppressed thyroid cancer cell viability, migration, invasion, proliferation, and induced cell apoptosis.

Numerous studies have demonstrated the oncogenic role of Smurf1 in human cells due to its contributory effects on aberrant degradation of tumor suppressor proteins [10,22, 23]. For instance, Xie et al. illustrated that Smurf1 was highly expressed in human lung cancer H1299 cells and promoted the ubiquitin dependent degradation of Kruppel-like factor 2 (KLF2), an essential protein in regulation of normal lung development and differentiation [24]. In the present study, we found that the expression levels of Smurf1 were higher in thyroid tumor tissues and thyroid cancer cells than those in the corresponding normal adjacent thyroid tissues and human normal thyroid cells. Knockdown of Smurf1 by transfection with sh-Smurf1 notably inhibited the SW579 and TPC-1 cell viability, migrations, invasion and proliferation, but remarkably increased cell apoptosis. The expression levels of Bax, CleavedCaspase 3, and Cleaved-Caspase 9 were also enhanced in SW579 and TPC-1 cells after transfection with sh-Smurf1. In contrast, transfection with pc-Smurf1 significantly increased the SW579. TPC-1, K1 and BCPAP cell viability, migrations, invasion and proliferation. These findings indicated that Smurf1 played important roles in the canceration of normal thyroid cells and up-regulation of Smurf1 promoted the growth and metastasis of thyroid cancer cells. In addition, these results were quite consistent with the previous study, which pointed out that Smurf1 remarkably promoted breast cancer cell migration and invasion [9].

Tumor metastasis is the common features of malignancy, which explains why surgical resection and adjuvant therapy can not completely cure cancers. KISS-1 has been found to play key roles in reducing metastasis of many cancers. The expression level of KISS-1 commonly decreases or completely losses in a variety of cancer cells through unclear mechanisms or pathways [25]. In this research, our results revealed that Smurf1 negatively regulated the protein levels of KISS-1 in thyroid cancer SW579 and TPC-1 cells, but not influenced the mRNA levels of KISS-1. Further experiments found that Smurf1 promoted the ubiquitination of KISS-1 in thyroid cancer cells, which suggested that Smurf1 enhanced the metastasis of thyroid cancer might by increasing the ubiquitin-dependent degradation of KISS-1 in thyroid cancer cells.

NF- $\kappa \mathrm{B}$ signaling pathway is one of the most indispensable cellular signaling pathways in both normal cells and cancer cells [26]. Chen et al. proved that KISS-1 up-regulation significantly inhibited the proliferation, migration, and invasion of human colorectal cancer HCT116 cells through inactivating NF- $\mathrm{BB}$ signaling pathway [27]. In addition, Cho et al. pointed out that KISS-1 inhibited the tumor necrosis factor-alpha (TNF- $\alpha$ )-induced breast cancer cell invasion via down-regulation of rashomologue A (RhoA)-mediated NF- $\kappa B$ activation [28]. In terms of thyroid cancer, a number of NF- $\kappa B$ signaling pathway inhibitors have been found to reduce the thyroid cancer cell proliferation and invasion, especially in combination with chemo- or radio-therapy [29]. In this study, the protein expression levels of p-p65 and p-IкB $\alpha$ were both decreased after up-regulation of KISS-1, which indicated that KISS-1 was involved in the regulation of NF- $\mathrm{KB}$ signaling pathway in thyroid cancer cells and over-expression of KISS-1 inhibited the activation of NF- $\kappa B$ signaling pathway in 
thyroid cancer cells. These results were consistent with the study of Morello et al., which demonstrated that the adenosine receptor A3 agonist (CI-IB-MECA) promoted the tumor necrosis factor-related apoptosis-inducing ligand (TRAIL)-induced thyroid cancer cell apoptosis through suppressing the NF- $\mathrm{kB}$ signaling pathway [30]. Furthermore, we also revealed that overexpression of KISS-1 suppressed thyroid cancer cell viability, migration, invasion and proliferation, and induced cell apoptosis. These results further confirmed the tumor suppressive roles of KISS-1 in thyroid cancer cells.

\section{Conclusion}

Our research verified that Smurf1 exerted important roles in thyroid cancer development by promoting thyroid cancer cell proliferation and metastasis. The ubiquitin-dependent degradation of KISS-1 induced by Smurf1 and the NF- $\kappa B$ signaling pathway activation might be involved in this process. This study not only expanded the understanding of Smurf1 and KISS-1 on thyroid cancer cell proliferation and metastasis, but also provided possible effective therapy targets and biomarkers for thyroid cancer treatment.

\section{Acknowledgements}

This study was funded by Technology Development Program of Jining in 2015. This study was approved by the Institutional Ethics Committee of the Jining No. 1 People's Hospital.

\section{Disclosure Statement}

The authors declare to have no competing interests.

\section{References}

1 Wojakowska A, Chekan M, Widlak P, Pietrowska M: Application of Metabolomics in Thyroid Cancer Research. Int J Endocrinol 2017;2015:258763.

2 Mallia N, Vassallo J, Pathways S: Pathogenesis of endocrine thyroid cancer. Malta Med J 2015;27:28-33.

3 Schmidt B, Davies L: The Rising Incidence of Thyroid Cancer: Contributions from Healthcare Practice and Biologic Risk Factors, in Mancino A, Kim L (eds): Management of Differentiated Thyroid Cancer. Springer, Cham, 2017.

-4 Riesco-Eizaguirre G, Santisteban P: ENDOCRINE TUMOURS: Advances in the molecular pathogenesis of thyroid cancer: lessons from the cancer genome. Eur J Endocrinol 2016;175:R203-R217.

5 Aberle H, Bauer A, Stappert J, Kispert A, Kemler R: beta-catenin is a target for the ubiquitin-proteasome pathway. EMBO J 2014;16:3797-3804.

6 Datta K, Suman S, Kumar S, Fornace AJ: Colorectal Carcinogenesis, Radiation Quality, and the UbiquitinProteasome Pathway. J Cancer 2016;7:174-183.

7 Ishii Y, Waxman S, Germain D: Targeting the ubiquitin-proteasome pathway in cancer therapy. Anticancer Agents Med Chem 2007; 7:359-365.

$>8$ Lee HL, Yi TG, Baek K, Kwon A, Hwang HR, Qadir AS, Park HJ, Woo KM, Ryoo HM, Kim GS: Tumor necrosis factor- $\alpha$ enhances the transcription of Smad ubiquitination regulatory factor 1 in an activating protein-1and Runx2-dependent manner. J Cell Physiol 2013;228:1076-1086.

-9 Kwon A, Lee HL, Woo KM, Ryoo HM, Baek JH: SMURF1 Plays a role in EGF-induced breast cancer cell migration and invasion. Mol Cells 2013;36:548-555.

10 Ke M, Mo L, Li W, Zhang X, Li F, Yu H: Ubiquitin ligaseSMURF1 functions as a prognostic marker and promotes growth and metastasis of clear cell renal cell carcinoma. FEBS Open Bio 2017;7:577-586. 


\section{Cellular Physiology Cell Physiol Biochem 2018;49:2047-2059

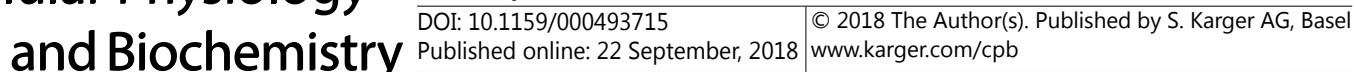

Yan et al.: Roles of Smurf1 in Thyroid Cancer Cells

11 Shin WJ, Cho YA, Kang KR, Kim JH, Hong SD, Lee JI, Hong SP, Yoon HJ: KiSS-1 expression in oral squamous cell carcinoma and its prognostic significance. APMIS 2016;124:291-298.

-12 Sanchezcarbayo M, Capodieci P, Cordoncardo C: Tumor suppressor role of KiSS-1 in bladder cancer: loss of KiSS-1 expression is associated with bladder cancer progression and clinical outcome. Am J Pathol 2003;162:609-617.

13 Makri A, Pissimissis N, Lembessis P, Polychronakos C, Koutsilieris M: The kisspeptin (KiSS-1)/GPR54 system in cancer biology. Cancer Treat Rev 2008;34:682-692.

$\$ 14$ Ji K, Ye L, Mason MD, Jiang WG: The Kiss-1/Kiss-1R complex as a negative regulator of cell motility and cancer metastasis (Review). Int J Mol Med 2013;32:747-754.

15 Savvidis C, Papaoiconomou E, Petraki C, Msaouel P, Koutsilieris M: The Role of KISS1/KISS1R System in Tumor Growth and Invasion of Differentiated Thyroid Cancer. Anticancer Res 2015;35:819-826.

16 Gao RJ, Wang NP, Hui YE, Gao QJ, Zhou Y, Duan HS: Expression and Clinical Significance of Tumor Suppressor Gene KiSS-1 in Papillary Thyroid Carcinoma. Chin J Bases Clin General Surg 2010;8:465-473.

17 Zhu H, Kavsak P, Abdollah S, Wrana JL, Thomsen GH: A SMAD ubiquitin ligase targets the BMP pathway and affects embryonic pattern formation. Nature 1999;400:687-693.

18 Jiang R, Zhang C, Liu G, Gu R, Wu H: MicroRNA-126 inhibits proliferation, migration, invasion and EMT in osteosarcoma by targeting ZEB1. J Cell Biochem 2017;118:3765-3774.

19 Wang J, Zhang Y, Weng W, Qiao Y, Ma L, Xiao W, Yu Y, Pan Q, Sun F: Impaired Phosphorylation and Ubiquitination by p70 S6 Kinase (p70S6K) and Smad Ubiquitination Regulatory Factor 1 (Smurf1) Promote Tribbles Homolog 2 (TRIB2) Stability and Carcinogenic Property in Liver Cancer. J Biol Chem 2013;288:33667-33681.

20 Mao QD, Zhang W, Zhao K, Cao B, Yuan H, Wei LZ, Song MQ, Liu XS: MicroRNA-455 suppresses the oncogenic function of HDAC2 in human colorectal cancer. Braz J Med Biol Res 2017;50:e6103.

-21 Ciechanover A: The ubiquitin-proteasome pathway: on protein death and cell life. EMBO J 1998;17:71517160.

22 Zhang Y, Wang C, Cao Y, Gu Y, Zhang L: Selective compounds enhance osteoblastic activity by targeting HECT domain of ubiquitin ligase Smurf1. Oncotarget 2016;18:50521-50533.

23 Lee MG, Jeong SI, Ko KP, Park SK, Ryu BK, Kim IY, Kim JK, Chi SG: RASSF1A Directly Antagonizes RhoA Activity through the Assembly of a Smurf1-Mediated Destruction Complex to Suppress Tumorigenesis. Cancer Res 2016;76:1847-1859.

-24 Xie P, Tang Y, Shen S, Wang Y, Xing G, Yin Y, He F, Zhang L: Smurf1 ubiquitin ligase targets Kruppel-like factor KLF2 for ubiquitination and degradation in human lung cancer H1299 cells. Biochem Biophys Res Commun 2011;407:254-259.

25 Mitchell DC, Abdelrahim M, Weng J, Stafford LJ, Safe S, Bar-Eli M, Liu M: Regulation of KiSS-1 metastasis suppressor gene expression in breast cancer cells by direct interaction of transcription factors activator protein-2alpha and specificity protein-1. J Biol Chem 2006;281:51-58.

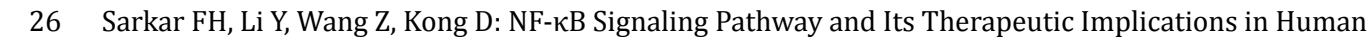
Diseases. Int Rev Immunol 2008;27:293-319.

-27 Chen S, Su X, Gao J, Han H, Chen Z, Lin S: [Suppression of Kiss-1 gene inhibits HCT116 human colorectal carcinoma cell migration in vitro via nuclear factor- $\kappa B$ signaling pathway]. Nan Fang Yi Ke Da Xue Xue Bao 2015;35:1643-1648.

28 Cho SG, Li D, Stafford LJ, Luo J, Rodriguez-Villanueva M, Wang Y, Liu M: KiSS1 suppresses TNFalpha-induced breast cancer cell invasion via an inhibition of RhoA-mediated NF-kappaB activation. J Cell Biochem 2010;107:1139-1149.

29 Pacifico F, Leonardi A: Role of NF-кB in thyroid cancer. Mole Cell Endocrinol 2010;321:29-35.

-30 Morello S, Sorrentino R, Porta A, Forte G, Popolo A, Petrella A, Pinto A: Cl-IB-MECA enhances TRAILinduced apoptosis via the modulation of NF-kappaB signalling pathway in thyroid cancer cells. J Cell Physiol 2010;221:378-386. 